\title{
Low-temperature investigations of ion-induced amorphisation in silicon carbide nanowhiskers under helium irradiation
}

\author{
$\underline{\text { E. Aradi }}^{*}$, J. Lewis-Fell, G. Greaves, S.E. Donnelly and J.A. Hinks

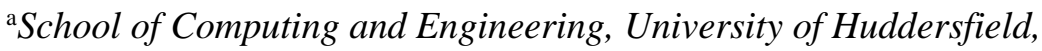 \\ Queensgate, Huddersfield, HD1 3DH, United Kingdom. \\ *Corresponding author: E.Aradi@hud.ac.uk
}

\section{Abstract}

The abundant free surface in nanoporous materials may play a major role in providing ideal sinks for the removal of radiation-induced defects. To study the response of these materials to radiation damage, 4H-silicon carbide ( $\mathrm{SiC}$ ) nanowhiskers (NWs) have been used to model individual ligaments of nanoporous SiC. Using in-situ transmission electron microscopy (TEM) with helium ion irradiation, a crystalline-to-amorphous transformation of the nanowhiskers was investigated as a function of irradiation dose and temperature. For a comparative analysis, the NWs were irradiated simultaneously alongside $\mathrm{SiC}$ thin foils (model systems for bulk-like $\mathrm{SiC}$ ) using $6 \mathrm{keV} \mathrm{He}$ ions at temperatures between 100 and $400 \mathrm{~K}$ and doses up to $50 \mathrm{dpa}$. Relatively-low swelling ( $8 \%)$ due to amorphisation was detected in the NWs compared to the foils $(\sim 14 \%)$ irradiated at room temperature. A relatively-high critical dose for amorphisation (5 dpa) was observed in the NWs for irradiations below room temperature compared to the foils ( $0.7 \mathrm{dpa})$. Amorphisation was completely avoided for NWs irradiated 
above $200 \mathrm{~K}$ - lower than the critical temperature in the foils which was $\sim 300 \mathrm{~K}$. The reduced swelling, higher critical-dose and lower critical-temperature for amorphisation exhibited by the NWs indicate an enhancement in radiation resistance over the foils.

Keywords: Nanoporous; Silicon carbide; nanowhiskers; amorphisation; in-situ TEM; radiation tolerance;

\section{Introduction}

The operational lifetimes of components in a nuclear reactor are primarily determined by the degradation of materials under radiation over extended periods of time. It is therefore necessary to carefully select structural materials capable of withstanding extreme conditions (high radiation damage levels, temperatures, thermo-mechanical stresses etc.). Silicon carbide ( $\mathrm{SiC}$ ) has been proposed for use as a first-wall material in fusion reactors, as an accident tolerant cladding material for currently light water reactors (LWRs), TRIstructure-ISOtropic (TRISO) fuel particle coatings for gas-cooled Generation IV fission reactors and as an inert matrix for the transmutation of plutonium [1], [2], [3], [4], [5], [6]. This is due to the fact that it possesses a high melting temperature (3000 K), high thermal conductivity $\left(490 \mathrm{Wm}^{-1} \mathrm{~K}^{-1}\right.$ at $\left.1273 \mathrm{~K}\right)$, low activation under neutron irradiation, chemical inertness and small neutron-capture cross-sections which collectively make it promising for applications in advanced nuclear systems [6]-[9].

Silicon carbide materials in fusion environments will be exposed to high radiation doses (up to $200 \mathrm{dpa}$ ) due to the large momentum transferred to the primary knock-on atoms by fast-moving neutrons at high temperatures $(>1273 \mathrm{~K})$ [3], [9]. This can initiate a cascade of subsequent atomic displacements 
resulting in the formation of point defect clusters that can grow to form extended defects such as dislocation loops. Helium gas will also be generated with rates up to 500 atomic parts per million (appm) of He being produced per year in the DEMOnstration (DEMO) power plant per full-power year from transmutation reaction processes [10]. This damage accumulation will accelerate the degradation of the material through processes such as hardening, embrittlement, creep and dimensional changes potentially leading to failure of the material under irradiation [10]-[13]. Amorphisation, one of the possible effects of radiation damage in ceramic materials, greatly impacts the mechanical properties of SiC by inducing significant swelling and substantial decreases in hardness [14]-[16].

Various strategies being investigated to produce potentially radiation-tolerant materials for use in nuclear environments involve the fabrication of nanostructured materials with either high densities of interfaces or grain boundaries to act as sinks for point defects, dislocation loops and impurity atoms. This can reduce their accumulation in the matrix and thus potentially improving the radiation tolerance [17]-[21].

Recent studies have shown that the large free surface area in nanoporous materials can serve as an unsaturable sink for radiation induced defects at which they can be continuously removed resulting in less accumulation and thus increased radiation tolerance compared to their bulk counterparts [22]-[25]. If novel approaches can be developed to design nanoporous a $\mathrm{SiC}$ material capable of withstanding the aforementioned extreme reactor environments, then coupled with the intrinsic superior properties of $\mathrm{SiC}$, this could lead to a breakthrough in the ability to tailor these materials to yield high radiation tolerance for the purposes of enhancing nuclear safety and realising fusion and Generation IV energy production. 
In this study, using in-situ transmission electron microscopy (TEM), we investigate the effect of He ion irradiation on 4H-SiC nanowhiskers (NWs) which act as a model system for nanoporous $\mathrm{SiC}$ and report on the crystalline to amorphous transition as a function of dose and irradiation temperature. The study compares the effect of radiation damage of the NWs with irradiation of $4 \mathrm{H}-\mathrm{SiC}$ thin foils by considering equal damage levels (in terms of displacements per atom) produced by incident ions in the NWs and the foils. The dose above which the material became amorphous will be referred to as the critical dose throughout the text 


\section{Experimental}

The SiC NWs were obtained from Novarials Corporation, USA (Product code: NovaWires-SiC-WSK). The average diameter of the NWs was about $60 \mathrm{~nm}$ with lengths up to $5 \mu \mathrm{m}$. To provide support and allow for side-by-side analysis during irradiation, the NWs were dispersed onto $4 \mathrm{H}-\mathrm{SiC}$ thin foils. The foils were prepared from $4 \mathrm{H}-\mathrm{SiC}$ wafers obtained from Cree Corporation, USA (Product code: W4NPE4C-Y200). Using an ultrasonic disc cutter, the wafer was cut into $3 \mathrm{~mm}$ discs which were mechanically polished to $\sim 100 \mu \mathrm{m}$ and dimpled to $\sim 50 \mu \mathrm{m}$ then ion-beam milled using a precision ion polishing system (PIPS) to a thickness range of $50-70 \mathrm{~nm}$ as described previously [26]. All the samples used were $4 \mathrm{H}-\mathrm{SiC}$ with $99.9 \%$ purity. Sample thickness was estimated using electron energy-loss spectroscopy (EELS) and thickness mapping was performed using energy-filtered TEM (EFTEM) taking the electron mean-free-path, $\lambda$, for $\mathrm{SiC}$ as $166 \mathrm{~nm}$ for $300 \mathrm{keV}$ electrons [16]. The thin foil was then dipped into NW powder resulting in adherence to the foil with some protruding from the edge of the thin region of the foil which could then be studied whilst avoiding potential electron and/or ion beam shadowing effects by the foil.

Irradiations were carried out between 100 and $400 \mathrm{~K}$ (which are low enough temperatures to supress any annealing effects and avoid bubble formation [27]) using $6 \mathrm{keV}$ He ions at a flux of $10^{14}$ ions.cm ${ }^{-2}$ $\mathrm{s}^{-1}$. In-situ TEM observations were performed using the Microscope and Ion Accelerator for Materials Investigations (MIAMI-2) system [28] located at the University of Huddersfield. MIAMI-2 consists of a $350 \mathrm{kV}$ ion accelerator coupled with a Hitachi H-9500 TEM in which the ion beam is incident at $18.7^{\circ}$ to the electron beam at the sample position. The microscope was operated at $300 \mathrm{kV}$ and the electron beam was turned off during ion irradiation to minimise synergistic effects between the electron and ion 
beams. The H-9500 TEM is capable of EELS and EFTEM measurements using a Gatan image filter (GIF Quantum SE Model 963). The EELS spectra were collected with a collection angle greater than $100 \mathrm{mrad}$ (no objective aperture condition) and using the TEM in imaging mode. A Gatan Model 636 cooling holder was used for the low temperature in-situ experiments. Further ex-situ analyses were carried out using a JEOL JEM-3010 TEM.

The Stopping and Range of Ions in Matter (SRIM) Monte Carlo code [29] was used to calculate the damage distribution for $6 \mathrm{keV} \mathrm{He}$ ions in SiC. Since SRIM estimates the damage in a planar target, an algorithm using MATLAB known as Ion Damage and RAnge in the Geometry Of Nanowires (IDRAGON) [30] was designed to account for the circular cross-sectional shape of a nanowire. The IDRAGON calculations were performed for $3000 \mathrm{He}$ ions at an energy of $6 \mathrm{keV}$, with a displacement energy of $35 \mathrm{eV}$ for $\mathrm{Si}$ and $21 \mathrm{eV}$ for $\mathrm{C}$ [31], and the target density to $3.21 \mathrm{~g} / \mathrm{cm}^{3}$ [32]. The $6 \mathrm{keV} \mathrm{He}$ energy ensures that the peak damage is placed close to the centre of a $60 \mathrm{~nm}$ diameter NW (average NW size used in this work) and in a $60 \mathrm{~nm}$ thick foil as shown in figure 1 . The number of displacements per atom (dpa) was calculated from the COLLISION.TXT files obtained from the "detailed calculations with full cascade" option using equation 1.

$$
d p a=\frac{D \emptyset}{\rho_{a t}}
$$

Where $D$ is the total displacements $(\mathrm{Si}$ vacancies $+\mathrm{C}$ vacancies + replacement collisions events) per unit length, $\phi$ is the total fluence and $\rho_{a t}$ the atomic number density of the material. 
Figure 1 (a) shows the results of SRIM calculations of a $60 \mathrm{~nm}$ foil (using conventional SRIM) and fig.

1 (b) for a $60 \mathrm{~nm}$ diameter NW (using the IDRAGON code) irradiated with $6 \mathrm{keV} \mathrm{He}$ ions showing the dpa as a function of depth at a fluence of $1 \times 10^{17}$ ions.cm ${ }^{-2}$.
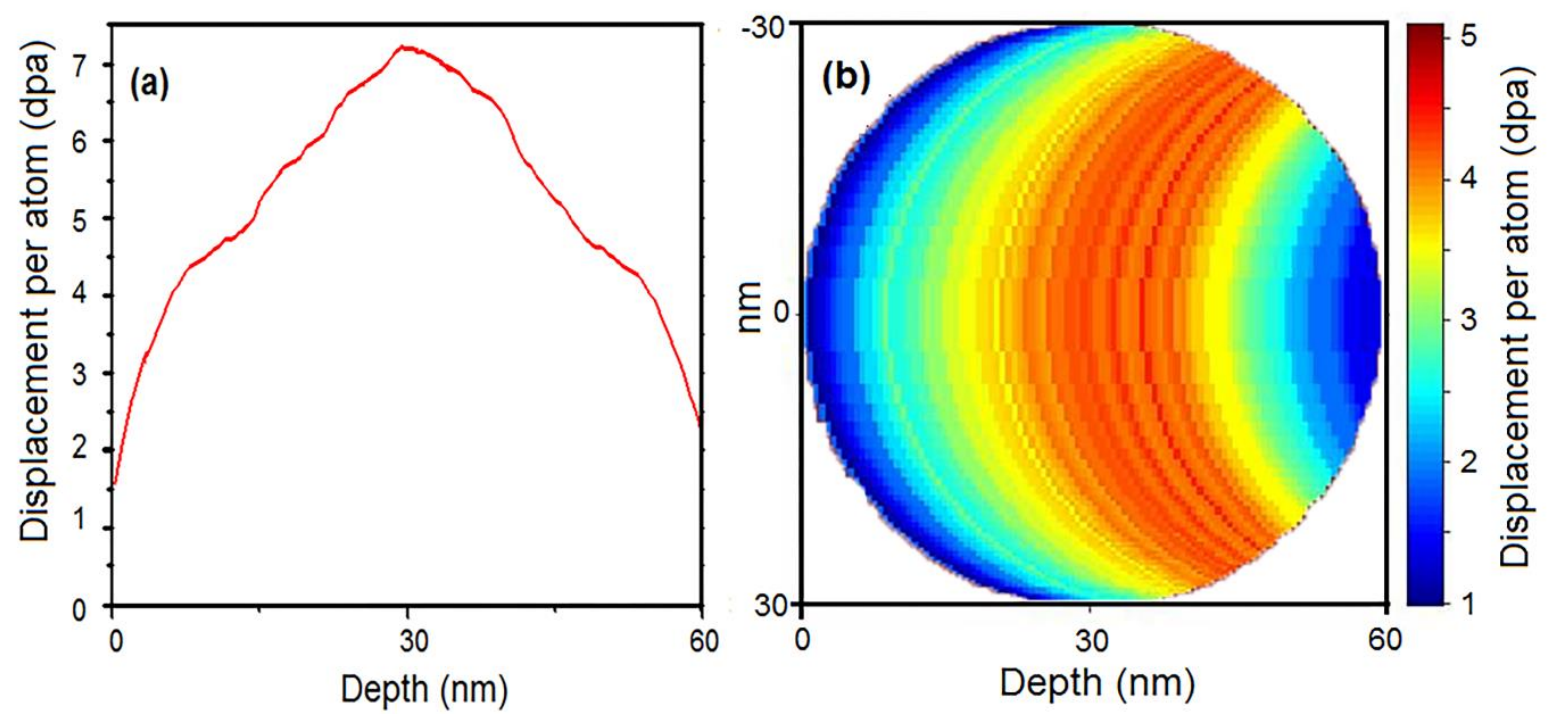

Figure 1: (a) SRIM calculations for the damage profile for a $60 \mathrm{~nm}$ thin foil and (b) SRIM calculations modified using the IDRAGON code for a circular cross-section of a cylindrical NW with 60 nm diameter irradiated with $6 \mathrm{keV}$ He showing the displacement per atom as a function of depth for the samples at a fluence of $1 \times 10^{17}$ ions. $\mathrm{cm}^{-2}$.

Because of the different geometries in the NWs and the foils, the average damage levels across the thickness of each will be different for the same fluence. To achieve the same damage of, for example, $0.05 \mathrm{dpa}$ in a $60 \mathrm{~nm}$ diameter NWs and $60 \mathrm{~nm}$ thickness foils when irradiated with $6 \mathrm{keV} \mathrm{He}$, the NW must be irradiated to a fluence of about $5 \times 10^{13}$ ions. $\mathrm{cm}^{-2}$ higher than that for the foils. With the in-situ TEM technique, these different fluences were targeted by imaging the NWs and foils after appropriate 
irradiation steps. For all the experiments reported in this study, $60 \pm 10 \mathrm{~nm}$ diameter NWs and $60 \pm 10$

$\mathrm{nm}$ thick regions in the foils were analysed.

\section{Results and discussion}

\subsection{Changes in $\mathrm{SiC}$ microstructure induced by room temperature irradiation}
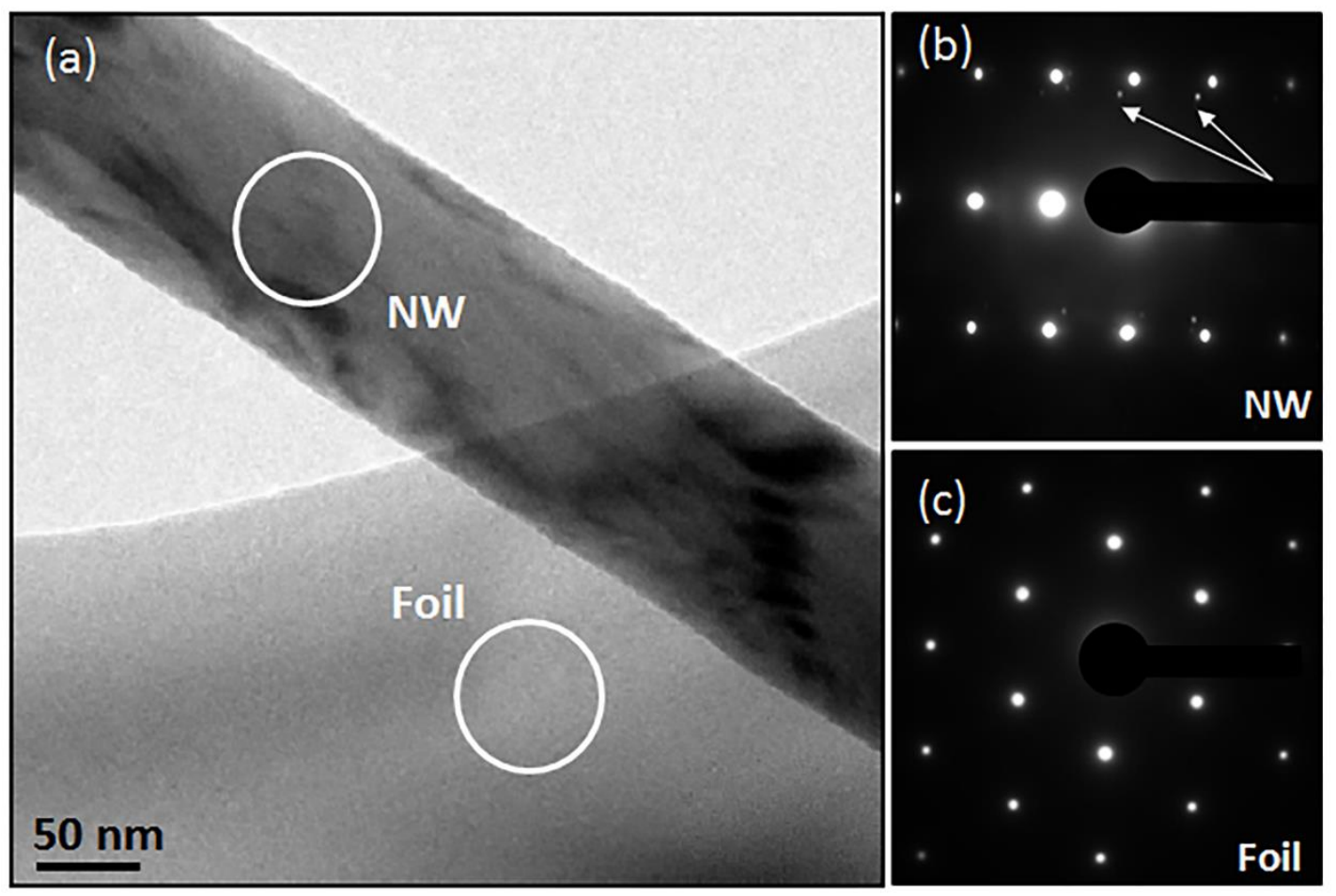

Figure 2 (a) BF-TEM image recorded at $200 \mathrm{~nm}$ underfocus showing a SiC NW attached to the thin

edge of the foil before irradiation. (b) The SADP acquired from the encircled region of the $N W$ shown in (a) orientated along the [011̄1] zone axis while (c) is the SADP acquired from the encircled region in the foil in (a) orientated along the [0001] zone axis. The SADP shows that foils were single crystalline while the NW contained twin crystals indicated by extra diffraction spots (arrowed in (b)) and confirmed via high resolution TEM (not shown). 
Figure 2 shows the BF-TEM image of a NW at the edge of a thin foil and the corresponding SADPs before irradiation. The SADPs show the [01111] and [0001] zone axes for the NW and foil obtained within $0.5^{\circ}$ of each other. The SADP of the NW in fig. 2 (b) was obtained near a twin boundary and it shows two sets of diffraction spots that resulted from the bicrystal structure in this region of the NW while fig. 2 (c) is the SADP of a single crystalline foil.

Figure 3 shows examples of sequential BF-TEM images for the damage process in a SiC NW and a foil irradiated with $6 \mathrm{keV} \mathrm{He}$ at room temperature as a function of dose. The insets show the corresponding SADPs at the respective doses. Figure 3 (a) features the NW imaged at a fluence of $2.4 \times 10^{16}$ ions. $\mathrm{cm}^{-2}$ (corresponding to $0.5 \mathrm{dpa}$ ) showing defect clusters resulting from the radiation damage. The SADP at this dose shows that the NW is still crystalline characterised by the lack of amorphous rings in the inset SADP. At the equivalent dose, fig. (d) shows BF-TEM of the foil with the SADP showing a coexistence of crystallinity with a developing amorphous phase evidence by faint rings. The change in diffraction contrast observed in the BF-TEM image of the NW at 1 dpa in fig 3 (b) is most likely due to dislocation loops, defect clusters and bend contours as the sample locally enters Bragg conditions during irradiation. The SADP at this dose (1 dpa) also shows an emerging amorphous rings together with diffraction spots for the NW. At higher fluences of $1.6 \times 10^{17}$ ions. $\mathrm{cm}^{-2}$ and $2.0 \times 10^{17}$ ions. $\mathrm{cm}^{-2}$ for the NW and foil (fig. 3 (c) and (f)), respectively, corresponding to $3.5 \mathrm{dpa}$, the SADP of the foil shows a complete loss of crystallinity evidenced by diffuse amorphous rings while the NW has the coexistence of both crystalline and amorphous phases. 

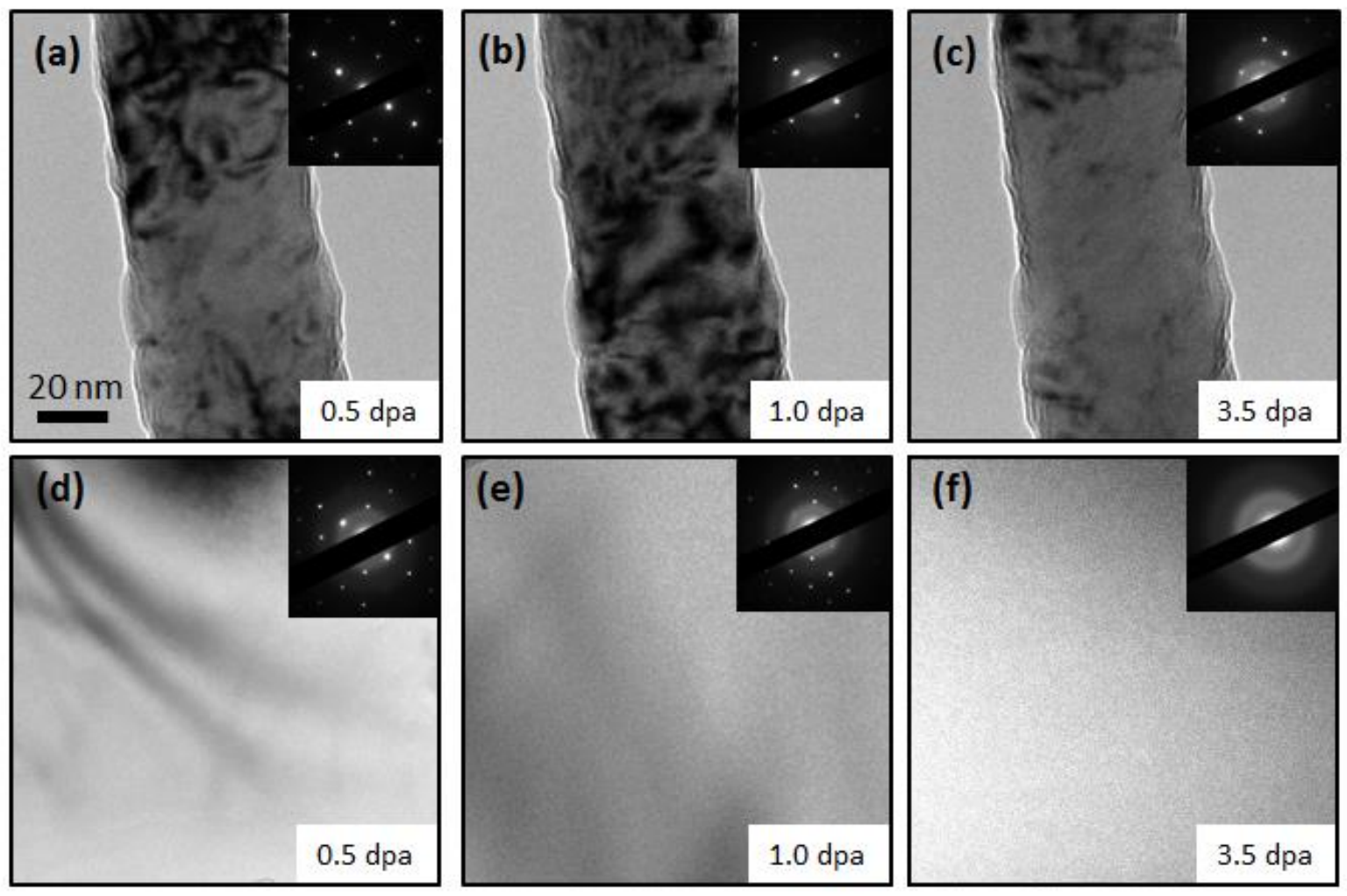

Figure 3: BF-TEM images of $(a-c)$ a SiC NW and $(d-f)$ foil irradiated with $6 \mathrm{keV} \mathrm{He}$ at room temperature and up to (a, d) $0.5 \mathrm{dpa},(b, e) 1.0 \mathrm{dpa}$ and $(c, f) 3.5 \mathrm{dpa}$. The insets show the corresponding SADPs. (Scale bar in (a) applies to all BF images.)

The nucleation and growth of amorphous regions in a material will be determined by how defects and defect clusters accumulate in regions of cascade overlap [33]. For both types of specimen, the initial irradiation step of $0.5 \mathrm{dpa}$ introduced only minor disorder due to minimum cascade overlap and thus the material remained predominantly crystalline (figs. 3 (a) and (d)). With increasing dose, the intensity of the crystalline reflections gradually reduced with the emergence of diffuse rings characteristic of amorphous material for both the NW (fig. 3(b-c)) and foil (fig. 3(d-e). With 
increasing dose there would have been further cascade overlap enabling small defect clusters to grow and coalesce to form denser clusters or amorphous regions detected by the SADP. A complete loss of long-range order was seen at $3.5 \mathrm{dpa}$ in the foil in contrast to the NW which was not observed to amorphise.

No bubble nucleation was observed in both the foils and NWs up to the final dose of 50 dpa equivalent to a fluence of $2.1 \times 10^{18}$ ions.cm ${ }^{-2}$ and $2.4 \times 10^{18}$ ions.cm ${ }^{-2}$, respectively, for these room-temperature irradiations. This dose was equivalent to He implantation concentration of 74 at. $\%$ and 77 at. $\%$ for the respective samples.

The NWs were irradiated with $6 \mathrm{keV} \mathrm{He}$ at room temperature to higher doses and fig. 4 shows an example of the BF-TEM, nano-beam diffraction patterns (NBDPs) and dark field (DF) images of a NW irradiated to a fluence of $2 \times 10^{18}$ ions. $\mathrm{cm}^{-2}$ corresponding to $46 \mathrm{dpa}$. The NBDPs were taken from different regions of the NW indicated by circles in the BF-TEM image in fig. 4 (a). The NBDP in fig. 4 (b) shows partial amorphisation of the NW indicated by a combination of diffuse rings and faint diffraction spots. In fig. 4 (c) taken from a different region on the same NW, the NBDP shows no visible diffraction spots demonstrating that complete amorphisation had occurred within this region. Figure 4 (d) shows the DF image for the NW acquired from the diffraction spot in dicated in (b) showing the crystalline pockets in the NW. This indicates that the entire NW was not fully amorphised with doses as high as $46 \mathrm{dpa}$. This result, i.e. where complete amorphisation of the NW was not achieved at up to 50 dpa but was at $\sim 4$ dpa in the foil, has been replicated in 12 separate experiments covering 65 different NWs and foil regions with diameters and thicknesses in the range of $60 \pm 10 \mathrm{~nm}$ in addition to the examples presented above. 

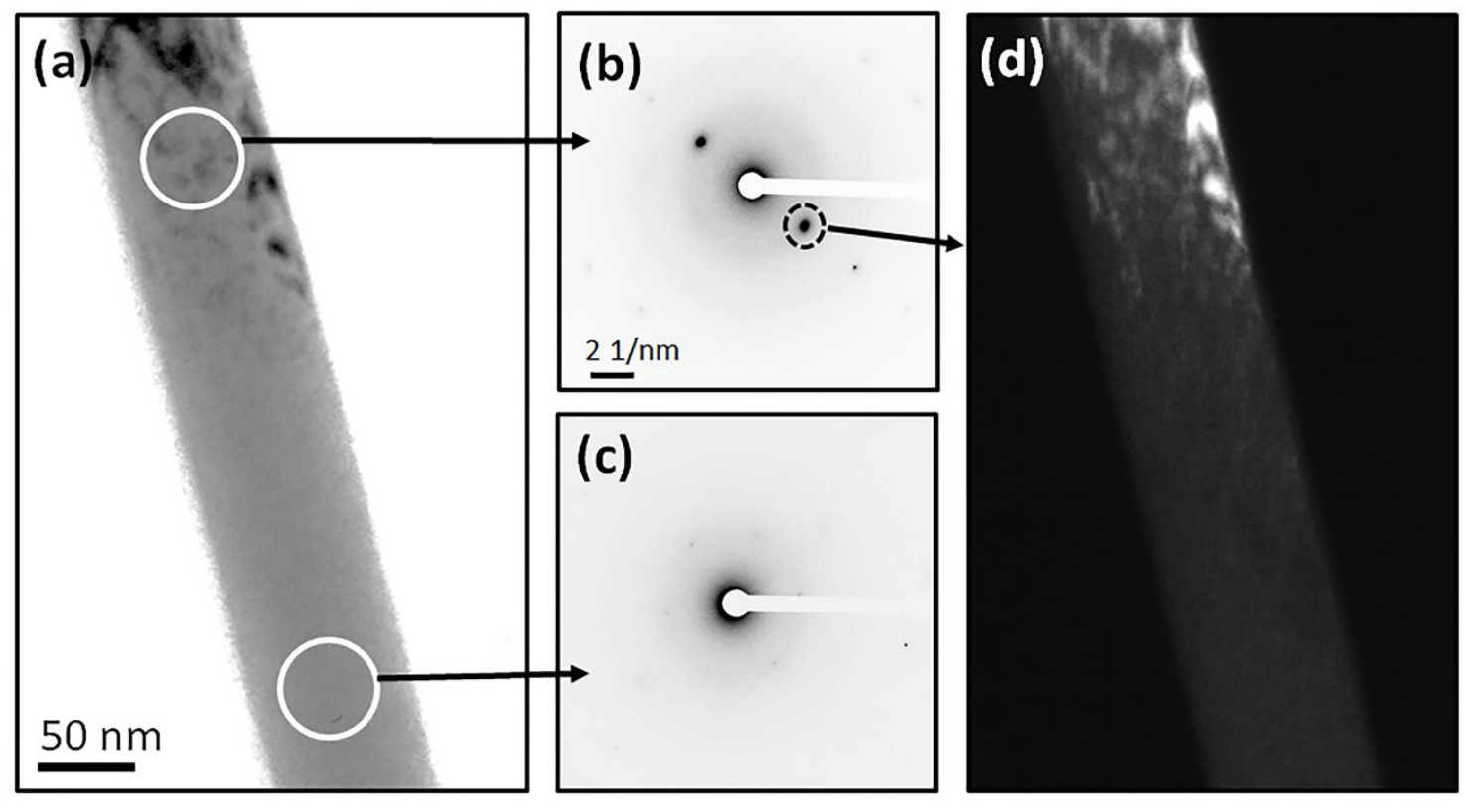

Figure 4: (a) BF-TEM image of a partially-amorphised NW irradiated to a dose of 46 dpa; (b and $c$ ) show the NBDPs taken from the indicated regions. The NBDP in (b) shows partial amorphisation whilst (c) shows complete amorphisation within this region. The DF-TEM image in $(d)$ was formed from the diffraction spot indicated in (b) showing regions with crystallinity.

Previous computer simulations and experiments have revealed that ion-induced amorphisation occurs due to $\mathrm{Si}-\mathrm{C}$ bond breaking followed by subsequent $\mathrm{Si}-\mathrm{Si} / \mathrm{C}-\mathrm{C}$ homonuclear bond formation and also due to cascade overlap during displacement spikes within the SiC network [16], [34], [35]. These $\mathrm{Si}$ and $\mathrm{C}$ defects increase the Gibbs free energy which can be reduced by a transition to an amorphous state [34]. Amorphisation in turn causes substantial lattice swelling in the material that subsequently affects its mechanical properties [7], [14], [36], [37]. 
Amorphisation of various $\mathrm{SiC}$ polymorphs has been reported for electron, neutron and ion irradiation at room temperature. Ishimaru et al. [38] using 200-300 keV electrons at room temperature observed a non-flux-dependant crystalline-to-amorphous transformation of $6 \mathrm{H}-\mathrm{SiC}$ with a subsequent transformation to amorphous silicon attributed to preferential displacement of $\mathrm{C}$ atoms. Inui et al. [39] reported electron-induced amorphisation at room temperature at $2 \mathrm{MeV}$ at a critical dose of $1.1 \mathrm{dpa}$ in $6 \mathrm{H}-\mathrm{SiC}$ while Jamison et al. [17] reported amorphisation of nanocrystalline 3C-SiC at a dose of 2.26 dpa after irradiation with $1.25 \mathrm{MeV}$ electrons at $173 \mathrm{~K}$. Snead et al. [40] reported complete amorphisation of $6 \mathrm{H}$ and $3 \mathrm{H}-\mathrm{SiC}$ after neutron irradiation to a dose of $2.5 \mathrm{dpa}$ at room temperature while Jin et al. [34] using molecular dynamics reported a neutron-induced crystalline to amorphous transition at a dose of $0.27 \mathrm{dpa}$ in $3 \mathrm{C}-\mathrm{SiC}$. Heavy ion irradiation has also been shown to cause amorphisation in $\mathrm{SiC}$ with the critical dose decreasing with increasing ion mass which has been attributed to an increase in the residual disorder per ion impact [16], [36], [41]-[47].

Closely related to the current work, He irradiation has been studied and reported to induce amorphisation in single crystalline SiC. Liu et al. [48] using $70 \mathrm{keV}$ He ions and Leclerc et al. [14] using $50 \mathrm{keV} \mathrm{He}$ ions at room temperature reported amorphisation critical doses of $1.1 \mathrm{dpa}$ and $1.3 \mathrm{dpa}$, respectively, whilst Harrison et al. [26] observed amorphisation of $4 \mathrm{H}-\mathrm{SiC}$ at room temperature at a dose of $1.5 \mathrm{dpa}$ after irradiation with $\mathrm{He}$ at $20 \mathrm{keV}$. Overall, there is a decrease in the critical amorphisation dose with increasing He ion energy reported in the literature. The 3.5 dpa average critical dose for $6 \mathrm{keV} \mathrm{He}$ irradiation of a SiC thin foils reported in the current work therefore fits well with this trend. 
Various studies have shown an increased resistance to amorphisation when $\mathrm{SiC}$ material has been tailored by modifying the size from bulk single crystal to nanosized structures. Imada et al. [41] reported an improved radiation tolerance in nano-engineered (NE) 3C-SiC compared to single-crystal 3C-SiC with an increased critical dose using $10 \mathrm{MeV}$ Au ions at room temperature. This was attributed to high twin-boundary and stacking-fault densities in the NE material acting as defect sinks thus aiding damage recovery [41]. Gosset et al. [49], on the other hand, using $4 \mathrm{MeV}$ Au ions reported a high critical dose in nanometric high density grain sized 3C-SiC compared to bulk $\mathrm{SiC}$ which was attributed to preferential energy deposition at the grain boundaries which modified the cascade structure aiding crystal healing. Taguchi et al. [50] observed amorphisation of SiC nanotubes after irradiating with 200 $\mathrm{keV} \mathrm{Si}$ ions to $2.9 \mathrm{dpa}$ at room temperature which was a higher dose compared to the dose for $\mathrm{Si}$ implanted bulk $\mathrm{SiC}(0.56 \mathrm{dpa}$ at $0.56 \mathrm{MeV})$ reported in [51]. Jamison et al. [17] using electron irradiation observed increased amorphisation resistance in nanocrystalline $\mathrm{SiC}$ compared to single crystal $\mathrm{SiC}$ which they attributed to a reduced energy barrier for $\mathrm{Si}$ interstitials and recombination reaction at grain boundaries.

Returning to the current work, complete amorphisation of the NWs with high doses (up to 50 dpa) was not achieved compared to complete amorphisation of the foils at fairly low doses ( 3.2 and $4.5 \mathrm{dpa})$ under identical energy and temperature conditions. These observations indicate a better resistance of the NWs to amorphisation as compared to the foils at room temperature. Various possible explanations have been proposed in this study to explain the increase in the critical dose observed in the NWs compared to the thin films at this temperature. It has been reported that defect production in nanostructured materials differs from bulk systems due to reduced dimensionality in one or more 
direction, thereby the energy dissipation following an ion impact will be affected [52]. At room temperature, self-interstitials and vacancies are immobile in $\mathrm{SiC}$ due to their high migration barriers (about $4 \mathrm{eV}$ and $7 \mathrm{eV}$ respectively, for $\mathrm{SiC}$ [53]). The small dimensions of the NWs will therefore be expected to reduce the effective thermal conductivity (i.e. 1D cooling where heat is dissipated in only one direction) which may give rise to higher local temperatures during the (fairly dilute in the case of He) thermal spike phase. This will have the effect of increasing the local temperature of the material and thus enhancing defect mobility. Conversely in the foil, the kinetic energy (KE) of the PKAs is dissipated through its large volume (3D cooling where heat is dissipated in all directions) reducing recombination as the defects experience less enhancement to their mobility.

The proximity of the surface also reduces the required migration distance of defects enabling recombination at the surface hence suppressing defect accumulation and subsequent amorphisation of the NW. Furthermore, molecular dynamics calculations by Sun et al. [46] showed that the long range order of bulk $\mathrm{SiC}$ decreases at a faster rate with increasing dose compared to that in nanowires under irradiation with $\mathrm{C}$ and $\mathrm{Si}$ ions at $5 \mathrm{keV}$ which they attributed to a complete cascade development in the bulk material but not in the NWs.

In addition to having a large-surface-area-to-volume ratio, the NWs used in the current work contained twinned crystals and therefore the twin boundaries will act as sinks which may have an enhancing effect on the capture and annihilation of interstitials and vacancies [41], [54]. With a higher probability of point defects annihilation at the surface and other interfaces, it is therefore more difficult to amorphise NWs compared to the foil with equal damage levels at room temperature resulting in better radiation tolerance. 


\subsection{Volume expansion at room temperature}

Figure 5 shows typical low-loss EELS spectra measured in the NWs and the foils before and after irradiation with $6 \mathrm{keV} \mathrm{He}$ at room temperature. The entire spectrum showing the zero loss peak is given in the supplementary data sheet. The spectra were recorded for irradiations carried out beyond the critical dose for the foils (at $10 \mathrm{dpa}$ ) and for the end dose (50 dpa) where large amorphous regions were observed in the NWs at room temperature. The spectra feature plasmon energy peaks located at $22.2 \pm 0.5 \mathrm{eV}$ and $21.3 \pm 0.5 \mathrm{eV}$ for the NWs before and after irradiation, respectively. For the foils, these were observed at $22.1 \pm 0.5 \mathrm{eV}$ and $20.8 \pm 0.5 \mathrm{eV}$, respectively. These measurements indicate a 0.9 and $1.3 \mathrm{eV}$ red shift of the plasmon energy peak as a result of the irradiation in the NW and foils, respectively.

Drude's model [55] describes how the plasmon energy, $E_{\mathrm{p}}$, lost by the electron beam when it generates a plasmon of frequency $\omega_{p}$ is proportional to the square root of the valence electron density, $n$, as shown in equation 2:

$$
E_{p}=\hbar \omega_{p}=\hbar\left(\frac{n e^{2}}{\epsilon_{o} m}\right)^{\frac{1}{2}}
$$

where $\hbar$ is the reduced Planck's constant, $m$ and $e$ are the effective mass and charge of the electron and $\varepsilon_{0}$ the permittivity of free space. Assuming minimal sputtering occurs during irradiation (supported by the in-situ observations), any displacement in the plasmon peak relates directly to the change in the atomic density of the material and hence its volume [55]. 

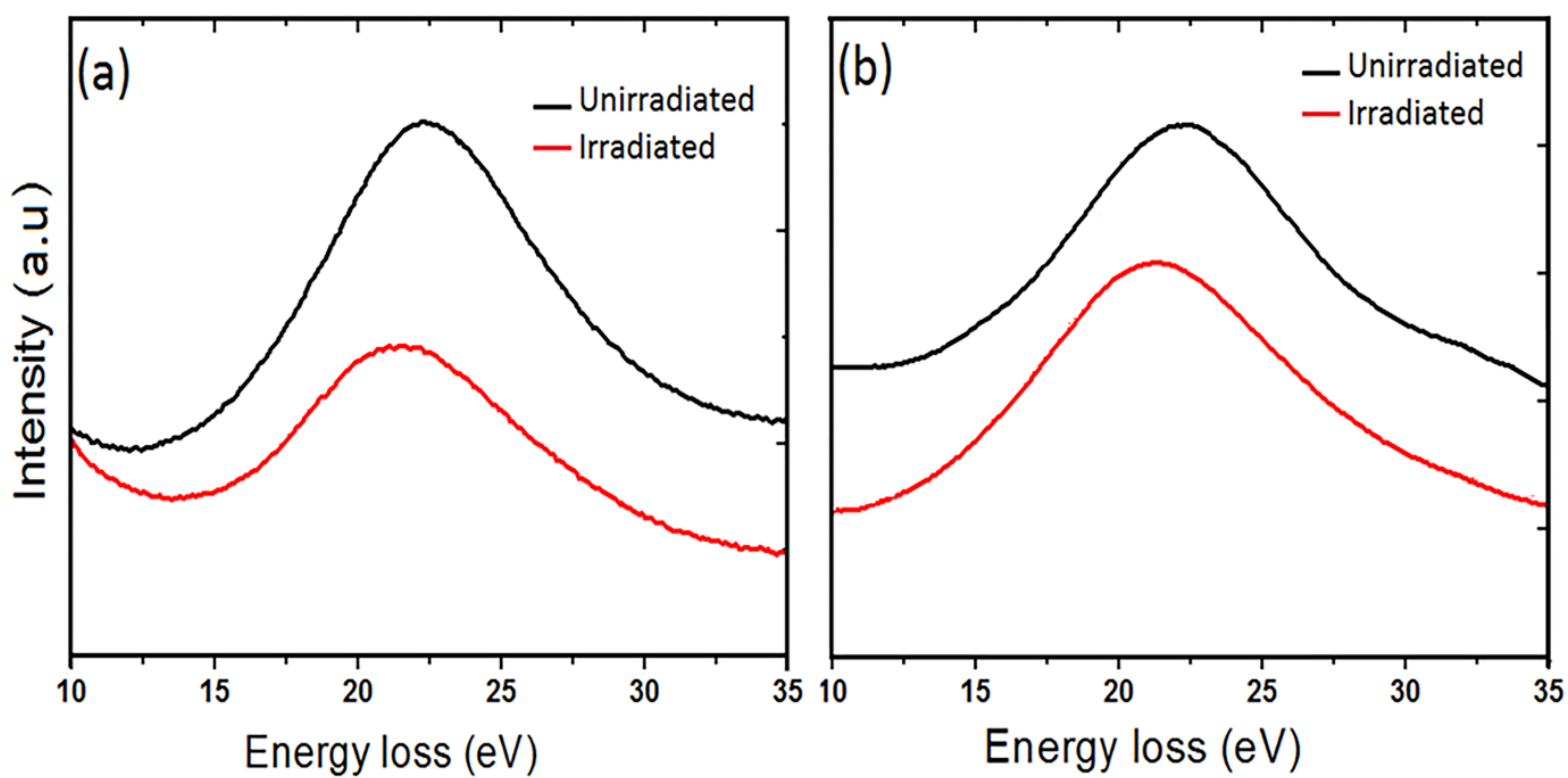

Figure 5: Low-loss EELS spectra obtained from (a) SiC NWs and (b) foil samples before and after irradiation with $6 \mathrm{keV} \mathrm{He}$ to 50 and 10 dpa, respectively, at room temperature.

A red shift in $E_{p}$ can therefore indicate a decrease in the atomic density of the material due to an outward spreading of the atoms resulting in a volumetric increase (i.e. swelling) of the material [56]. The volumetric change in the material as a result of amorphisation can then be predicted by taking measurements of the plasmon energy peak before and after irradiation using equation 3 [50], [55],

$$
\begin{aligned}
\frac{\Delta V}{V_{1}} & =\frac{V_{2}-V_{1}}{V_{1}} \\
& =\left[\frac{E_{P 1}}{E_{P 2}}\right]^{2}-1
\end{aligned}
$$

where $V$ is the volume interacting with the electron beam and $E_{\mathrm{p}}$ is the plasmon energy of the material with the subscripts 1 and 2 denoting before and after irradiation, respectively. Following equation 3, the average volume changes were estimated to be $7.6 \%$ for the NWs irradiated to 50 dpa and $13.8 \%$ for the foils irradiated to 10 dpa showing that the volume expansion in the foils was higher than the NWs 
for the room temperature irradiations. The density of amorphous $\mathrm{SiC}$ is about $12 \%$ less than that of crystalline material which is likely the major explanation for the $\sim 14 \%$ swelling observed in the foils after irradiation. The $7.6 \%$ volume expansion in the partially-amorphised NWs also falls below the typical swelling range of $\mathrm{SiC}$ bulk that has been reported in literature to range between $13-15 \%$ for electron [35], ion [15], [37] and neutron [47] irradiation experiments, indicating a better tolerance to radiation induced swelling in NWs.

\subsection{Temperature dependence on amorphisation}

Figure 6 shows the relationship between the critical dose and the implantation temperature for the NWs and foils. The data recorded in fig. 6 was obtained for 18 different experiments with a total of 84 NWs and at 40 different regions in the foil samples. These observations were quantified by observing the changes in the diffraction patterns of the samples with increasing dose and the critical dose was determined when the sample became completely amorphous. 


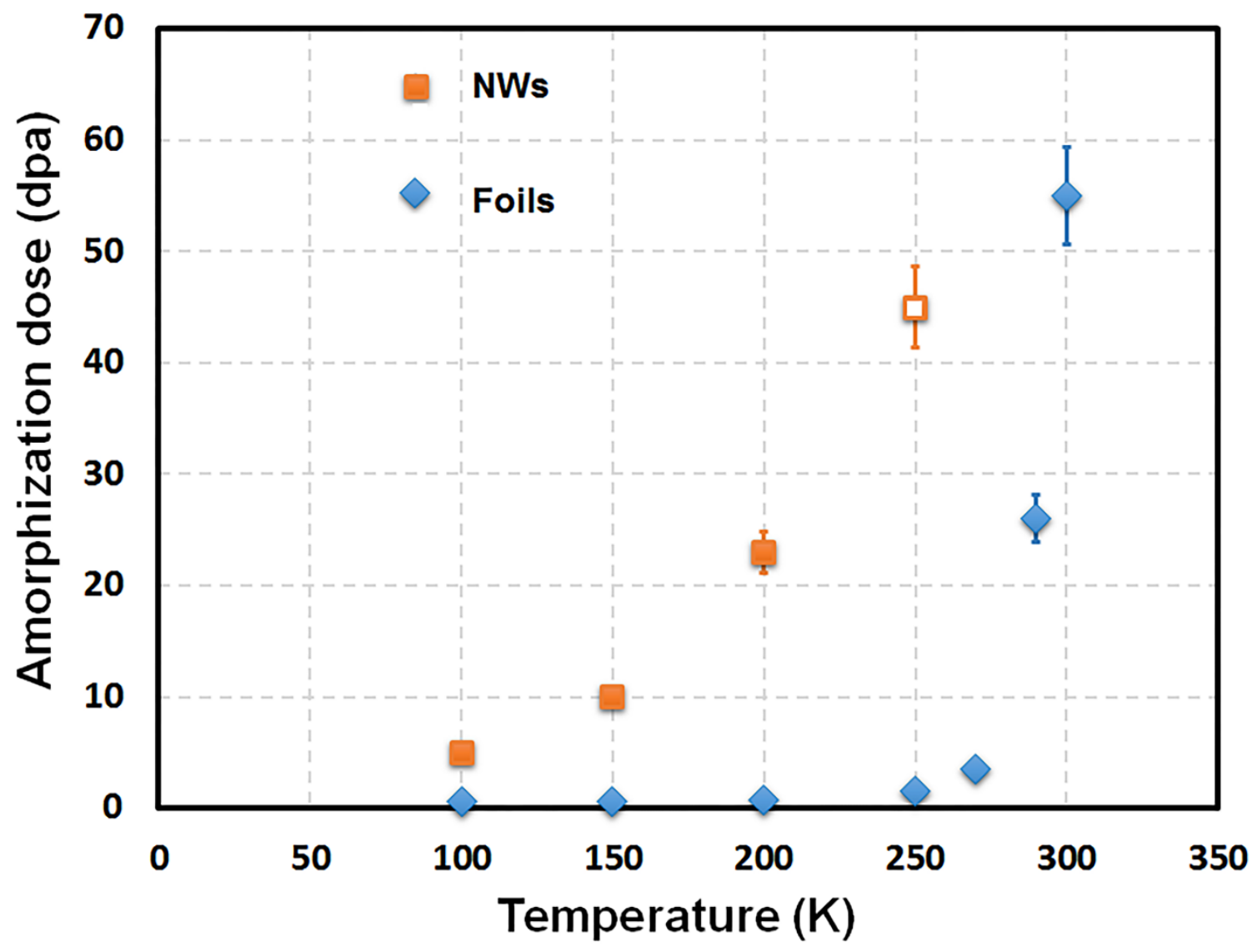

Figure 6. Relationship between the irradiation temperature and average critical dose for amorphisation for $N W$ and foils irradiated with $6 \mathrm{keV} \mathrm{He.} \mathrm{The} \mathrm{open} \mathrm{symbol} \mathrm{shows} \mathrm{the} \mathrm{data} \mathrm{for} \mathrm{coexistence} \mathrm{of}$ amorphous and crystalline phases at the maximum dose reached.

From the graph in fig. 6, the NWs showed higher average critical doses compared to the foils for all temperatures - for instance $5 \mathrm{dpa}$ and $0.6 \mathrm{dpa}$ at $100 \mathrm{~K}$ and 23 and $0.7 \mathrm{dpa}$ at $200 \mathrm{~K}$ for the NWs and foils, respectively. The NW data show an increase in the amorphisation dose with temperature when the samples were irradiated between 100 and $200 \mathrm{~K}$. At $250 \mathrm{~K}$, the NWs were partially disordered exhibiting both amorphous and crystalline phases as observed in the DP for an average dose of $45 \mathrm{dpa}$ (indicated by the open symbol) and remained crystalline for irradiations carried out at $270 \mathrm{~K}$ and beyond doses $>50 \mathrm{dpa}$. The experimental data for the foils shows a discernibly different plot compared with the NWs over the same temperature range showing a relatively-constant mean 
critical dose ( $\sim 0.7 \mathrm{dpa})$ with increasing temperature between 100 and $200 \mathrm{~K}$. Between 250 and 270 $\mathrm{K}$ there is a gradual increase in the critical dose and at $290 \mathrm{~K}$ the critical dose increases rapidly with temperature. This indicates that recovery is dependent on a thermally-activated exponential process which becomes effective above $200 \mathrm{~K}$. Above $300 \mathrm{~K}$ the material remained completely crystalline even for irradiation to high doses (>50 dpa).

Several studies have reported a very small change in critical dose with increase in temperature for various single-crystal $\mathrm{SiC}$ polymorphs for temperatures below $277 \mathrm{~K}$ and above this temperature the critical dose increased rapidly [37], [57], [58]. This temperature is defined as the critical temperature for amorphization, $T_{c}$ above which the material remains crystalline [32], [34]. This temperature is consistent with the $\sim 290 \mathrm{~K}$ critical temperature reported here for the foil samples.

With increasing irradiation temperature, the rate of damage annealing increases and therefore a higher dose is needed to induce amorphisation in both the foils and NWs (with a much higher average dose (almost 5 times) needed for the NW at $100 \mathrm{~K}$ ). At the critical temperature, there is a balance between the defect production and recovery. Above the critical temperature, the rate of damage recovery exceeds the production through dynamic annealing and hence the material remains crystalline as the point defects have enough thermal energy to overcome the migration barrier enhancing annihilation.

These observations suggest that, as would be expected, higher temperatures increase recovery for both materials but that it is sufficiently effective to supress amorphisation at a lower temperatures in the NWs. As discussed above, the abundant surface in the NWs increases the probability of diffusion processes resulting in a migrating defect arriving at the surface and annihilating. A 
proportion of vacancies will be located close enough to the surface to feel its image force; therefore, even at low temperatures where they cannot normally diffuse there is an increased chance of annihilation within a few nanometers of the surface. For the foil samples, because of the greater average migration distance to the nearest surface, there will be a lower probability of recombination creating a higher density of defects at low temperature aiding amorphisation at lower doses.
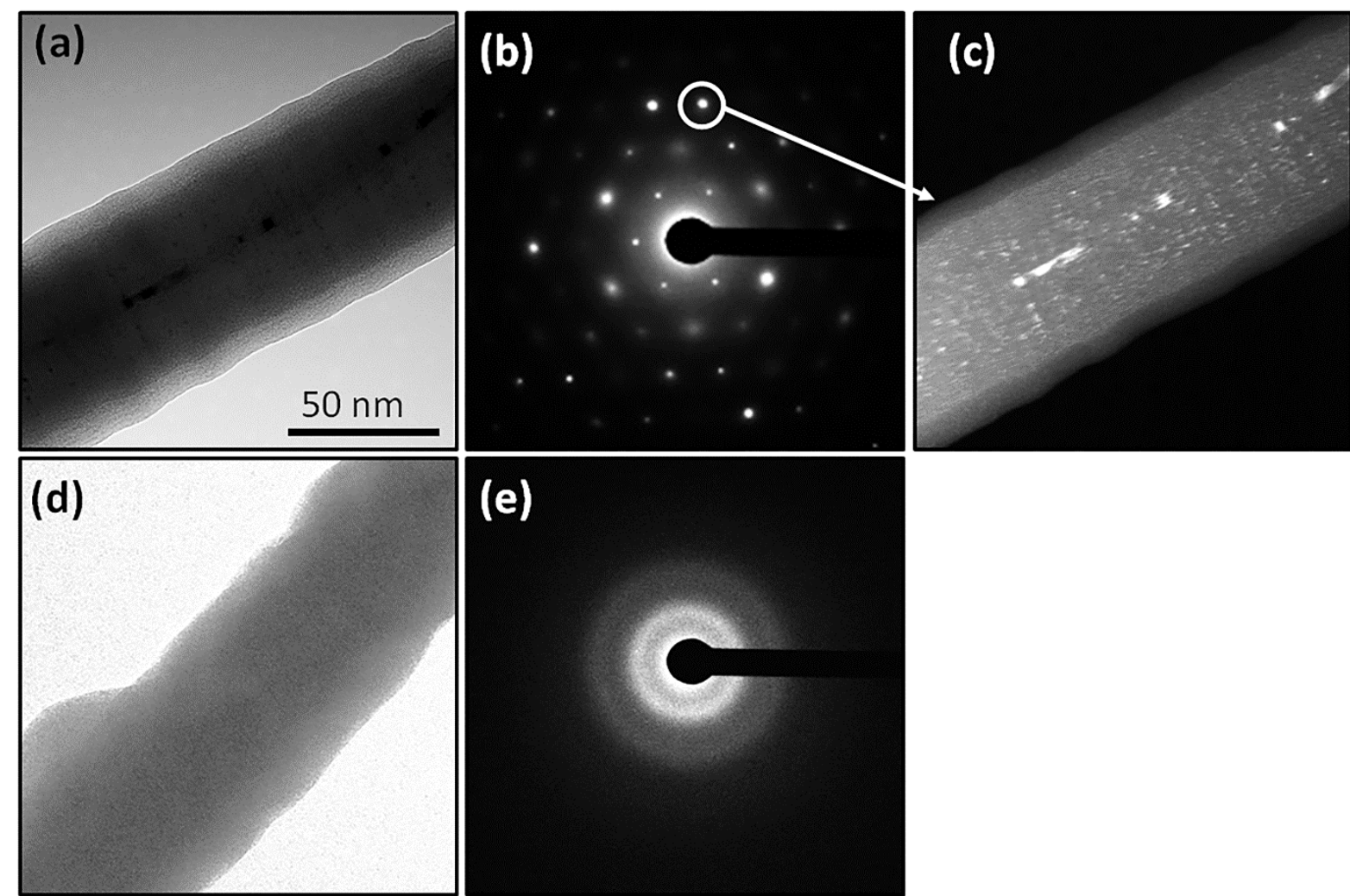

Figure 7 (a-c): BF, SADP and DF images for SiC NWs irradiated with $6 \mathrm{keV}$ He to a dose of 30

dpa at $300 \mathrm{~K}$, respectively, and (d-f) BF, SADP and DF images for another $N W$ irradiated to a dose of 10 dpa at $100 \mathrm{~K}$, respectively.

Figure 7 show examples of two different NWs irradiated with $6 \mathrm{keV}$ He at 300 and $100 \mathrm{~K}$. Figure 7 (a) shows the presence of defect clusters or small loops seen as 1-3 nm black spots. The SADP in fig 7 (b) shows diffraction spots characteristic of crystalline $\mathrm{SiC}$ in the [111] zone axis while the DF in (c) shows 
the defect clusters as white dots, all indicating that the NWs remained crystalline despite the high irradiation dose used at this temperature. Figure 7 (d) shows no diffraction contrast while the SADP in (e) shows rings characteristic of a completely amorphous material.

\section{Conclusions}

This work compared the changes in the microstructure of SiC NWs and foils under $6 \mathrm{keV} \mathrm{He}$ ion irradiation at temperatures that allow a crystalline to amorphous transition in $\mathrm{SiC}$ (100-300 K). The following points give a summary of the findings from this study:

- Complete amorphisation was not realized in the NW for room temperature irradiation for doses up to $50 \mathrm{dpa}$, while the foil samples completely amorphised at an average dose of $3.5 \mathrm{dpa}$.

- The NWs showed a high resistance to amorphisation characterised by higher critical amorphisation doses for irradiation below room temperature.

- The overall swelling in the foil was twice as high as the swelling in NW for room temperature irradiations.

- Irradiation temperature played an important role in amorphisation. For temperatures above 250 $\mathrm{K}$, the NWs remained crystalline even at high doses while amorphisation was avoided at temperatures above $300 \mathrm{~K}$ in the foil samples.

At temperatures known to induce amorphisation in $\mathrm{SiC}, \mathrm{SiC}$ NWs showed a high resistance which was associated with two processes: the proximity to the surface that aided diffusion of interstitials to the surface and the subsequent recombination with vacancies near the surface that acts as an unsaturable 
sink, potentially removing point defects and secondly enhancing local annealing during irradiation that stimulated defect migration to the surface.

Studies on the use of nanoporous materials with an aim of fabricating the material for use in advanced nuclear reactor are still limited. However, using in-situ TEM ion irradiation, this study has shown that SiC NWs, as a model system for ligaments of nanoporous SiC, exhibits high amorphisation resistance as one aspect of superior radiation tolerance. This knowledge is important in understanding the role of defect-surface interactions in the creation of a form of $\mathrm{SiC}$ with greatly enhanced radiation resistance for potential applications in nuclear reactors.

\section{Acknowledgements}

This work was supported by EPSRC under grant numbers EP/E017266/2 and EP/M028283/1. The author would also like to appreciate Dr Anamul H. Mir and Dr R. W. Harrison for useful discussions.

\section{Conflict of interest}

The authors have no conflict of interest to disclose. 


\section{References}

[1] T. Hinoki et al., Silicon Carbide and Silicon Carbide Composites for Fusion Reactor Application, Mater. Trans., 54, (2013), 472476. https://doi.org/10.2320/matertrans.MG201206

[2] D. Blagoeva and S. G. De Vicente, Feature Article Current status and prospects of $\mathrm{SiC} \mathrm{f} / \mathrm{SiC}$ for fusion structural applications, J. Euro. Cer. Soc., 33, (2013), 1577-1589.

\section{https://doi.org/10.1016/0920-3796(95)80047-2}

[3] C. Chen et al., Dose dependence of helium bubble formation in nano-engineered $\mathrm{SiC}$ at $700^{\circ} \mathrm{C}$, J. Nucl. Mater., 472, (2016), 153-160. https://doi.org/10.1016/j.jnucmat.2016.01.029

[4] E. E. Bloom, S. J. Zinkle, and F. W. Wiffen, Materials to deliver the promise of fusion power Progress and challenges, J. Nucl. Mater., 329-333, (2004) 12-19. https://doi.org/10.1016/S0920-3796(01)00181-8

[5] A. R. Raffray et al., Design and material issues for high performance SiCf/SiC-based fusion power cores, Fusion Eng. Des., 55, (2001) 55-95.

[6] Y. Katoh, L. L. Snead, I. Szlufarska, and W. J. Weber, Radiation effects in SiC for nuclear structural applications, Curr. Opin. Solid State Mater. Sci., 16, (2012) 143-152. https://doi.org/10.1016/j.cossms.2012.03.005

[7] M. David et al., He implantation into 4H-SiC, Physc. Stat. Sol. A., 206, (2009) 1916-1923. http:// DOI 10.1002/pssa.200881468

[8] A. Misra, M. J. Demkowicz, X. Zhang, and R. G. Hoagland, The radiation damage tolerance of Ultra-High Strength Nanolayered Composites stability under ion, Jom, 59, (2007), 3-6.

[9] R. H. Jones et al., Promise and challenges of $\mathrm{SiCf} / \mathrm{SiC}$ composites for fusion energy applications, J. Nucl. Mater., 307-311, (2002), 1057-1072. https://doi.org/10.1016/S0022$\underline{3115(02) 00976-5}$ 
[10] W. J. Weber, W. Jiang, and S. Thevuthasan, Accumulation, dynamic annealing and thermal recovery of ion-beam-induced disorder in silicon carbide, 177, (2001), 26-30.

https://doi.org/10.1016/S0168-583X(00)00542-5

[11] C. J. Mchargue and J. M. Williams, Ion implantation effects in silicon carbide, Nuc. Inst. Meth. Phys. Res. 81, (1993), 889-894. https://doi.org/10.1016/0168-583X(93)90703-9

[12] E. Friedland, K. Gärtner, T. T. Hlatshwayo, N. G. Van Der Berg, and T. T. Thabethe, Influence of radiation damage on xenon diffusion in silicon carbide, Nucl. Inst. Methods Phys. Res. B, 332, (2014), 415-420. https://doi.org/10.1016/j.nimb.2014.02.109

[13] E. Friedland, Investigation of amorphisation energies for heavy ion implants into silicon carbide at depths far beyond the projected ranges, Nucl. Inst. Methods Phys. Res. B, 391, (2017), 10-13. https://doi.org/10.1016/j.nimb.2016.11.025

[14] S. Leclerc, A. Decímy, M. F. Beaufort, C. Tromas, and J. F. Barbot, Swelling of SiC under helium implantation, J. Appl. Phys., 98, (2005), 11. https://doi.org/10.1063/1.2137441

[15] H. Zang et al., Investigation of swelling induced by heavy ion and neutron irradiation in SiC, J. Nucl. Mater., 433, (2013), 378-381. https://doi.org/10.1016/j.jnucmat.2012.10.029

[16] J. Li et al., "Evolution of amorphisation and nanohardness in $\mathrm{SiC}$ under Xe ion irradiation," J. Nucl. Mater., 454, (2014),173-177. https://doi.org/10.1016/j.jnucmat.2014.07.036

[17] L. Jamison, M. J. Zheng, S. Shannon, T. Allen, D. Morgan, and I. Szlufarska, Experimental and ab initio study of enhanced resistance to amorphisation of nanocrystalline silicon carbide under electron irradiation, J. Nucl. Mater., 445, (2014), 181-189. https://doi.org/10.1016/j.jnucmat.2013.11.010

[18] M. Rose, A. G. Balogh, and H. Hahn, Instability of irradiation induced defects in nanostructured materials, Nucl. Instruments Methods Phys. Res. Sect. B Beam Interact. with Mater. Atoms, 127-128, (1997), 119-122. https://doi.org/10.1016/S0168-583X(96)00863-4 
[19] N. Nita, R. Schaeublin, and M. Victoria, Impact of irradiation on the microstructure of nanocrystalline materials, J. Nucl. Mater., 329-333, (2004), 953-957.

https://doi.org/10.1016/j.jnucmat.2004.04.058

[20] I. J. Beyerlein, A. Caro, M. J. Demkowicz, N. A. Mara, A. Misra, and B. P. Uberuaga, Radiation damage tolerant nanomaterials, Mater. Today, 16, (2013) 443-449. https://doi.org/10.1016/j.mattod.2013.10.019

[21] I. J. Beyerlein, M. J. Demkowicz, A. Misra, and B. P. Uberuaga, Defect-interface interactions, Prog. Mater. Sci., 74, (2015), 125-210. https://doi.org/10.1016/j.pmatsci.2015.02.001

[22] E. M. Bringa et al., Are nanoporous materials radiation resistant?, Nano Lett., 12, (2012), 3351-3355. https://doi.org/10.1021/nl201383u

[23] J. Li, H. Wang, and X. Zhang, A Review on the Radiation Response of Nanoporous Metallic Materials, Jom, (2018). https://doi.org/10.1007/s11837-018-3111-x

[24] E. Aradi et al., Enhanced Radiation Tolerance of Tungsten Nanoparticles to He Ion Irradiation, Nanomaterials, 8, (2018), 1052. https://10.3390/nano8121052

[25] N. J. Briot, M. Kosmidou, R. Dingreville, K. Hattar, and T. J. Balk, In situ TEM investigation of self-ion irradiation of nanoporous gold, J. Mater. Sci., 54, (2019), 7271-7287. https://10.1007/s10853-019-03385-z

[26] R. W. Harrison, S. Ebert, J.A. Hinks, and S.E. Donnelly, Damage microstructure evolution of helium ion irradiated $\mathrm{SiC}$ under fusion relevant temperatures, J. Eur. Ceram. Soc., 8, (2018), 3718-3726. https://doi.org/10.1016/j.jeurceramsoc.2018.

[27] J. Roy, S. Chandra, S. Das, and S. Maitra, Oxidation behaviour of silicon carbide - A review, Rev. Adv. Mater. Sci., 38, (2014), 29-39. https://10.1016/j.jnucmat.2007.05.016

[28] G. Greaves, A. H. Mir, R. W. Harrison, M. A. Tunes, S. E. Donnelly, and J. A. Hinks, New Microscope and Ion Accelerators for Materials Investigations (MIAMI-2) system at the University of Huddersfield, Nucl. Instruments Methods Phys. Res. Sect. A Accel. 
Spectrometers, Detect. Assoc. Equip., 931, (2019), 37-43.

https://doi.org/10.1016/j.nima.2019.03.074

[29] J. F. Ziegler, M. D. Ziegler, and J. P. Biersack, SRIM - The stopping and range of ions in matter (2010), Nucl. Instruments Methods Phys. Res. Sect. B Beam Interact. with Mater. Atoms, 268, (2010), 1818-1823. https://doi.org/10.1016/j.nimb.2010.02.091

[30] O. Camara et al., Shape Modification of Germanium Nanowires during Ion Irradiation and Subsequent Solid-Phase Epitaxial Growth, 5, (2018), 1-9. https://doi.org/10.1002/admi.201800276

[31] H. L. Heinisch, L. R. Greenwood, W. J. Weber, and R. E. Williford, Displacement damage cross sections for neutron-irradiated silicon carbide, 311, (2002), 895-899. https://doi.org/10.1016/S0022-3115(02)00962-5

[32] Y. Zhang et al., Effects of implantation temperature on damage accumulation in Al-implanted 4H - SiC, 4012, (2005). https://doi.org/10.1063/1.1666974

[33] F. Gao and W. J. Weber, "Cascade overlap and amorphisation in 3C-SiC: Defect accumulation, topological features, and disordering," Phys. Rev. B - Condens. Matter Mater. Phys., vol. 66, (2002), 1-10. https:// doi.org/10.1103/PhysRevB.66.024106

[34] E. Z. Jin and L. S. Niu, "Crystalline-to-amorphous transition in silicon carbide under neutron irradiation,"Vaccum, 86, (2012), 917-923. https://doi.org/10.1016/j.vacuum.2011.05.013

[35] S. Muto and T. Tanabe, "Local structures and damage processes of electron irradiated $\alpha-\mathrm{SiC}$ studied with transmission electron microscopy and electron energy-loss spectroscopy," $J$. Appl. Phys., 93, (2003), 3765-3775. https://doi.org/10.1063/1.1555673

[36] J. F. Barbot et al., Strain Build-Up, Swelling and Stacking Fault Formation in Implanted 4H SiC, Mater. Sci. Forum, 717-720, (2012), 485-488. DOI: 10.1016/j.jnucmat.2011.04.022

[37] Y. Katoh, H. Kishimoto, and A. Kohyama, Low Temperature Swelling in Beta-SiC Associated 
with Point Defect Accumulation, Mater. Trans., 43, (2002), 612-616.

\section{https://doi.org/10.2320/matertrans.43.612}

[38] M. Ishimaru, I. T. Bae, and Y. Hirotsu, Electron-beam-induced amorphisation in SiC, Phys. Rev. B - Condens. Matter Mater. Phys., 68, (2003), 1-4. https://link.aps.org/doi/10.1103/PhysRevB.68.144102

[39] H. Inui, H. Mori, and H. Fujita, Electron-irradiation-induced crystalline to amorphous transition in $\alpha$-Sic single crystals, 61, (1990), 107-124. http// 10.1080/13642819008208655

[40] C. M. Parish et al., Helium sequestration at nanoparticle-matrix interfaces in helium and heavy ion irradiated nanostructured ferritic alloys, J. Nucl. Mater., 483, (2017), 21-34.

\section{https://doi.org/10.1016/j.jnucmat.2016.10.038}

[41] K. Imada, M. Ishimaru, H. Xue, Y. Zhang, S. C. Shannon, and W. J. Weber, AMORPHISATION resistance of nano-engineered SiC under heavy ion irradiation, J. Nucl. Mater., 478, (2016), 310-314. https:// 10.1016/j.jnucmat.2016.06.031

[42] F. Gao, D. Chen, W. Hu, and W. J. Weber, Energy dissipation and defect generation in nanocrystalline silicon carbide, Phys. Rev. B - Condens. Matter Mater. Phys., 81, (2010), 1-8. https:// 10.1103/PhysRevB.81.184101

[43] W. J. Weber and F. Gao, Irradiation-induced defect clustering and amorphisation in silicon carbide, J. Mater. Res., 25, (2010), 2349-2353. https://doi.org/10.1557/jmr.2010.0292

[44] W. J. Weber, L. M. Wang, and N. Yu, The irradiation-induced crystalline-to-amorphous phase transition in $\alpha-S i C$, Nucl. Instruments Methods Phys. Res. Sect. B Beam Interact. with Mater. Atoms, 116, (1996), 322-326. https://10.1557/jmr.2010.0292

[45] C. Liu et al., Evolution of small defect clusters in ion-irradiated 3C-SiC: Combined cluster dynamics modeling and experimental study, Acta Mater., 125, (2017), 377-389. https://10.1016/j.actamat.2016.12.020

[46] L. Sun, C. Lan, S. Zhao, and J. Xue, Self-irradiation of thin SiC nanowires with low-energy 
ions : a molecular, J. Phys. Appl. Phys. 45, (2012), 135403. https://doi.org/10.1088/0022$\underline{3727 / 45 / 13 / 135403}$

[47] L. L. Snead, S. J. Zinkle, J. C. Hay, and M. C. Osborne, amorphisation of SiC under ion and neutron irradiation, Nucl. Instruments Methods Phys. Res. Sect. B Beam Interact. with Mater. Atoms, 141, (1998), 123-132. https://doi.org/10.1016/S0168-583X(98)00085-8

[48] M. Liu et al., Investigation of the damage behavior in CVD SiC irradiated with $70 \mathrm{keV} \mathrm{He}$ ions by NEXAFS, Raman and TEM, J. Eur. Ceram. Soc., 37, (2017) 1253-1259. https://doi.org/10.1016/j.jeurceramsoc.2016.11.046

[49] D. Gosset, A. Audren, Y. Leconte, L. Thomé, I. Monnet, and N. Herlin-Boime, Structural irradiation damage and recovery in nanometric silicon carbide, Prog. Nucl. Energy, 57, (2012), 52-56. https://doi.org/10.1016/j.pnucene.2011.12.013

[50] T. Taguchi, S. Yamamoto, and H. Ohba, Ion irradiation-induced novel microstructural change in silicon carbide nanotubes, Acta Mater., 154, (2018), 90-99.

https://doi.org/10.1016/j.actamat.2018.05.030

[51] T. Taguchi, S. Yamamoto, K. Kodama, and H. Asaoka, Synthesis of heterostructured SiC and C-SiC nanotubes by ion irradiation-induced changes in crystallinity, Carbon., 95, (2015), 279285. https://doi.org/10.1016/j.carbon.2015.08.056

[52] A. V. Krasheninnikov and K. Nordlund, Ion and electron irradiation-induced effects in nanostructured materials, J. Appl. Phys., 107, (2010). https://doi.org/10.1063/1.3318261

[53] H. Jiang, L. He, D. Morgan, P. M. Voyles, and I. Szlufarska, Radiation-induced mobility of small defect clusters in covalent materials, Phys. Rev. B, 94, (2016)1-5. https://link.aps.org/doi/10.1103/PhysRevB.94.024107

[54] F. W. Schapink, Comment on 'coherent annealing twin boundaries as vacancy sinks, Acta Metall., 12, (1964), 1297. DOI: 10.1016/0001-6160(64)90115-4 
[55] W. Jiang, C. M. Wang, W. J. Weber, M. H. Engelhard, and L. V. Saraf, Direct determination of volume changes in ion-beam-irradiated SiC, J. Appl. Phys., 95, (2004), 4687-4690.

https://doi.org/10.1063/1.1690102

[56] C. M. Wang, Microstructural features of Al-implanted 4H - SiC, J. Mater. Res. Soc. 18, (2003), 772-779. https://doi.org/10.1557/JMR.2003.0107

[57] C. H. Chen et al., Irradiation-induced microstructural change in helium-implanted single crystal and nano-engineered SiC, 453, (2014), 280-286.

https://doi.org/10.1016/j.jnucmat.2014.07.020

[58] H. Inui, H. Mori, and A. Suzuki, Electron-irradiation-induced crystalline-to-amorphous transition in $\beta$-SiC single crystals, Phil. Mag. B, 61, (2016), 37-41. 